THE AsTROPHYSICAL JouRNAL, 329:418-424, 1988 June 1

(C) 1988. The American Astronomical Society. All rights reserved. Printed in U.S.A.

\title{
LARGE MOLECULES IN DIFFUSE INTERSTELLAR CLOUDS
}

\author{
S. LEPP AND A. DALgaRno \\ Harvard-Smithsonian Center for Astrophysics \\ E. F. VAN Dishoeck \\ Princeton University Observatory \\ AND \\ J. H. BLACK \\ Steward Observatory \\ Received 1987 August 31; accepted 1987 November 25
}

\begin{abstract}
The effects of the presence of a substantial component of large molecules on the chemistry of diffuse molecular clouds are explored, and detailed models of the $\zeta$ Persei and $\zeta$ Ophiuchi clouds are constructed. The major consequence is a reduction in the abundances of singly charged atomic species. The long-standing discrepancy between cloud densities inferred from rotational and fine-structure level populations and from the ionization balance can be resolved by postulating a fractional abundance of large molecules of $1 \times 10^{-7}$ for $\zeta$ Persei and $6 \times 10^{-7}$ for $\zeta$ Ophiuchi. If the large molecules are polycyclic aromatic hydrocarbons (PAH) containing $\sim 50$ carbon atoms, they contain $1 \%$ of the carbon in $\zeta$ Persei and $7 \%$ in $\zeta$ Ophiuchi. Other consequences of the possible presence of PAH molecules are discussed.

Subject headings: interstellar: abundances - interstellar: molecules - molecular processes
\end{abstract}

\section{INTRODUCTION}

Large molecules and in particular polycyclic aromatic hydrocarbons (PAH) have been suggested as the sources of the broad infrared emission features in nebulae (Duley and Williams 1981; Léger and Puget 1984; Allamandola, Tielens, and Barker 1985) and of the diffuse interstellar absorption bands (Léger and d'Hendecourt 1985; van der Zwet and Allamandola 1985; Crawford, Tielens, and Allamandola 1985). If present in dense clouds at the level suggested for nebulae, where the large molecules may contain $2 \%$ or $3 \%$ of the interstellar carbon, they modify the chemistry of dense clouds substantially (Lepp and Dalgarno 1988). If present in diffuse clouds at a comparable fractional abundance, large molecules will cause changes in the predicted compositions. We demonstrate here that the ratios of successive ionization stages of atomic systems that do not react with hydrogen are sensitive to the abundances of large molecules and that upper limits to and useful estimates of the fractional abundance of large molecules in diffuse clouds can be derived from measurements of the relative abundances of neutral and singly ionized constituents.

Models of the composition of the diffuse molecular clouds lying toward $\zeta$ Persei, $\zeta$ Ophiuchi, $\chi$ Ophiuchi, and $o$ Persei have been constructed by van Dishoeck and Black (1986). We supplement their chemistry by postulating the presence of a component of large molecules (LM) which we take to have a chemistry like that of the polycyclic aromatic hydrocarbons.

\section{CHEMISTRY OF LARGE MOLECULES}

We adopt a characteristic ionization potential for LM of 6.8 $\mathrm{eV}$ and a characteristic electron affinity of $3 \mathrm{eV}$ (Omont 1986; Duley 1986; d'Hendecourt and Léger 1987). For the photoionization and photodetachment processes

$$
\begin{aligned}
\mathrm{LM}+h v & \rightarrow \mathrm{LM}^{+}+e, \\
\mathrm{LM}^{-}+h v & \rightarrow \mathrm{LM}+e,
\end{aligned}
$$

we adopt cross sections of $10^{-16} \mathrm{~cm}^{2}$. The resulting unshielded interstellar photoionization and photodetachment rates are $1.5 \times 10^{-8} \mathrm{~s}^{-1}$ and $6 \times 10^{-8} \mathrm{~s}^{-1}$, respectively, for the standard interstellar radiation field (see Draine 1978; van Dishoeck and Black 1986). To calculate the dependence of the rates on depth into a cloud, we follow the procedures of Roberge, Dalgarno, and Flannery (1981) with their grain models 2 and 3. We fitted the depth dependence by a bi-exponential form (see van Dishoeck and Dalgarno 1984).

The distribution of large molecules among $\mathrm{LM}^{+}, \mathrm{LM}$, and $\mathrm{LM}^{-}$is determined by the balance between photoionization and photodetachment and recombination

$$
\mathrm{LM}^{+}+e \rightarrow \mathrm{LM}
$$

and attachment

$$
\mathrm{LM}+e \rightarrow \mathrm{LM}^{-} .
$$

We adopted from Omont (1986) the recombination and attachment rate coefficients for molecules containing $50^{\circ}$ atoms. The values are $5.7 \times 10^{-6}(100 / T)^{1 / 2} \mathrm{~cm}^{3} \mathrm{~s}^{-1}$ and $7.7 \times 10^{-7} \mathrm{~cm}^{3}$ $\mathrm{s}^{-1}$, respectively, where $T$ is the temperature.

Figure 1 shows the calculated abundances of $\mathrm{LM}^{+}, \mathrm{LM}$, and $\mathrm{LM}^{-}$with depth into a cloud of uniform density $n_{\mathrm{H}}=200$ $\mathrm{cm}^{-3}$, electron fraction $n_{e} / n_{\mathrm{H}}=(1-3) \times 10^{-4}$ and temperature $50 \mathrm{~K}$ with visual extinction $A_{V}=1$ for grain model 2 in the standard interstellar radiation field. In such a cloud, the concentrations of $\mathbf{L M}$ and $\mathrm{LM}^{-}$are comparable and both are significantly larger than the concentration of $\mathrm{LM}^{+}$. The total electron density decreases with increasing large molecule abundance. This, in turn, causes the density ratios $\mathrm{LM} / \mathrm{LM}^{-}$and $\mathrm{LM}^{+} / \mathrm{LM}$ to increase as illustrated in Figure 1.

The negative ions $\mathrm{LM}^{-}$react rapidly with the positive ions $\mathrm{X}^{+}$in a process of mutual neutralization

$$
\mathrm{X}^{+}+\mathrm{LM}^{-} \rightarrow \mathrm{X}+\mathrm{LM}
$$



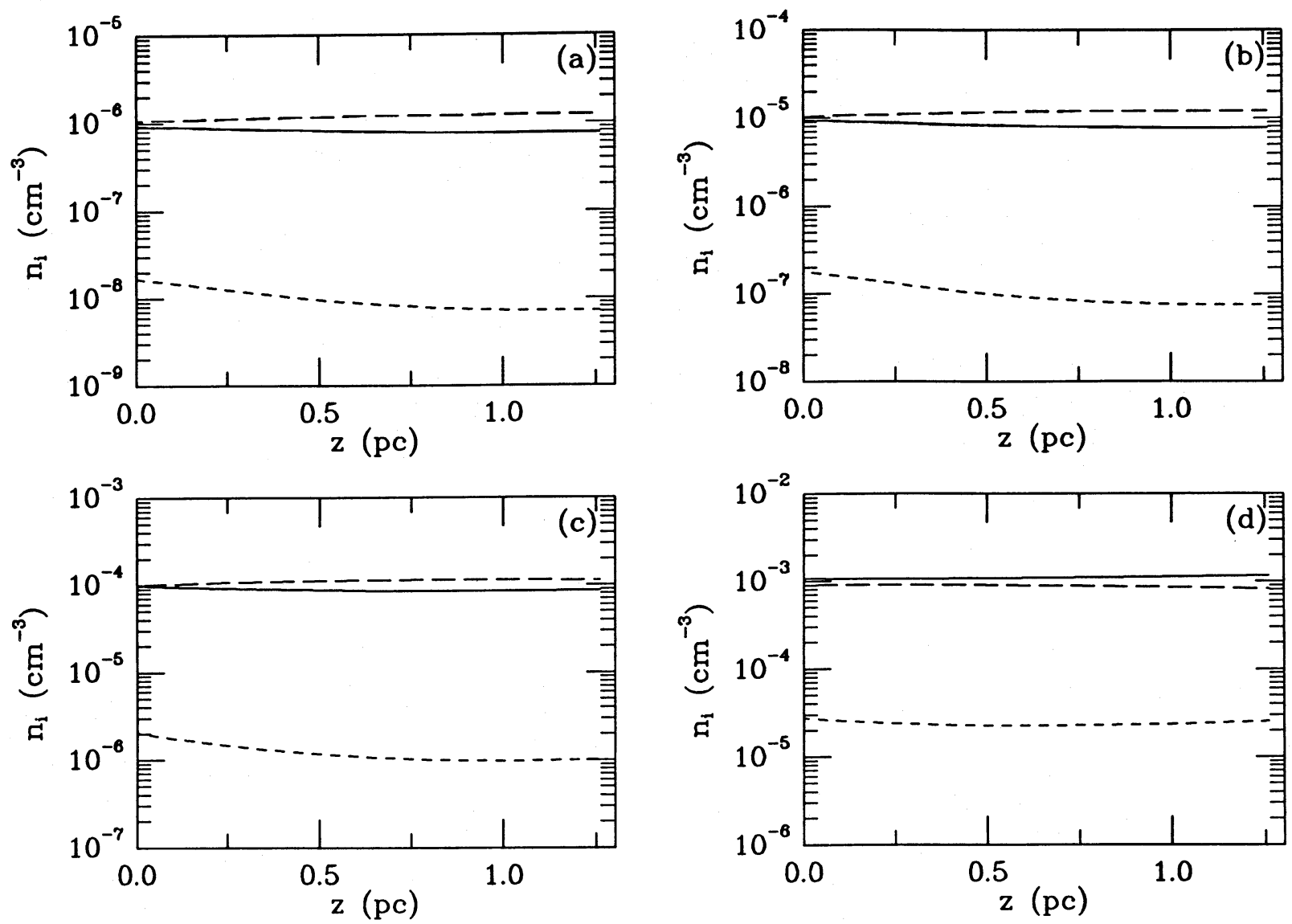

Fig. 1.-Depth variations of the concentrations of LM (solid curves), $\mathrm{LM}^{-}$(long dashed curves), and $\mathrm{LM}^{+}$(short dashed curves) in a reference model of a diffuse cloud. This uniform model has $n_{\mathrm{H}}=200 \mathrm{~cm}^{-3}, T=50 \mathrm{~K}$, and $A_{V}=1.0$ mag. Panels $(a),(b),(c)$, and $(d)$ show results for LM abundances of $x_{\mathrm{LM}}=10^{-8}, 10^{-7}, 10^{-6}$ and $10^{-5}$, respectively. When $x_{\mathrm{LM}} \geq 10^{-6}$, the effect of LMs on the overall ionization balance is large enough to produce a noticeable decrease in the electron density. This is accompanied by corresponding increases in the $\mathrm{LM} / \mathrm{LM}^{-}$and $\mathrm{LM}^{+} / \mathrm{LM}^{-}$density ratios.

(Omont 1986; Lepp and Dalgarno 1987). Positive ions also undergo charge transfer with neutral molecules

$$
\mathrm{X}^{+}+\mathrm{LM} \rightarrow \mathrm{X}+\mathrm{LM}^{+}
$$

if the ionization potential of $\mathrm{X}$ is greater than that of LM. If it is less, the reaction

$$
\mathrm{X}^{+}+\mathrm{LM} \rightarrow \mathrm{XH}^{+}+\mathrm{LM},
$$

in which a hydrogen atom is extracted from $\mathrm{LM}$ is assumed to occur. The molecular ions then undergo dissociative recombination

$$
\mathrm{XH}^{+}+e \rightarrow \mathrm{X}+\mathrm{H}
$$

at a rate coefficient assumed to equal $10^{-6} T^{-1 / 2} \mathrm{~cm}^{3} \mathrm{~s}^{-1}$.

In our further discussions we assume that the large molecules are polycyclic aromatic hydrocarbons (PAH).

The chemistry of PAH molecules is uncertain. We assume that the loss of atoms and molecules from the gas phase by attachment to PAH in any of its forms is small (Omont 1986). Duley and Williams (1986) have argued that reactions of PAH molecules with $\mathrm{H}, \mathrm{O}$, and $\mathrm{N}$ atoms are rapid and consequently destroy PAH on time scales less than $10^{5}$ yr whereas Omont (1986) suggests that reactions occur more slowly, primarily at radical sites on PAHs from which $\mathrm{H}$ atoms have been ejected by photodissociation. We write the rate coefficients for

$$
\begin{aligned}
& \mathrm{PAH}+\mathrm{O} \rightarrow \mathrm{PAH}+\mathrm{CO}, \\
& \mathrm{PAH}+\mathrm{N} \rightarrow \mathrm{PAH}+\mathrm{CN},
\end{aligned}
$$

as $10^{-10} f_{R} \mathrm{~cm}^{3} \mathrm{~s}^{-1}$ where $f_{R}$ is an efficiency factor related to the fraction of radical sites on the periphery of the PAH (van Dishoeck and Black 1988). For diffuse clouds $f_{R} \approx 0.1$. We assume that reaction of atomic carbon with PAH forms a special $\mathrm{C}$ site that is not part of the lattice skeleton

$$
\mathrm{PAH}+\mathrm{C} \rightarrow \mathrm{PAH}^{\prime},
$$

and that subsequent reactions with atomic hydrogen

$$
\mathrm{PAH}^{\prime}+\mathrm{H} \rightarrow \mathrm{PAH}+\mathrm{CH}_{4}
$$

form $\mathrm{CH}_{4}$, which can be released by photolysis. The effective rate coefficient for the sequence (11) and (12) is $10^{-10} f_{R}(1$ $\left.-f_{R}\right) / 4 \mathrm{~cm}^{3} \mathrm{~s}^{-1}$ (van Dishoeck and Black 1988). We assume further that $\mathrm{O}, \mathrm{N}$, and $\mathrm{C}$ react with $\mathrm{PAH}^{-}$to release the electron and liberate the saturated molecules $\mathrm{H}_{2} \mathrm{O}, \mathrm{NH}_{3}$, and $\mathrm{CH}_{4}$, respectively, with rate coefficients of $10^{-9} f_{R}\left(1-f_{R}\right) / m$ $\mathrm{cm}^{3} \mathrm{~s}^{-1}$, where $m$ is the number of hydrogen atoms in the liberated molecule. For reaction with $\mathrm{H}$, we suppose that $\mathrm{H}_{2}$ is 
formed,

$$
\mathrm{PAH}^{-}+\mathrm{H} \rightarrow \mathrm{PAH}+\mathrm{H}_{2}+e .
$$

The effect of equation (13) may be subsumed into the efficiency factor $y_{f}$ (van Dishoeck and Black 1986) for the formation of $\mathrm{H}_{2}$ by recombination on grain surfaces. Note that if each of the reactions (9)-(12) were accompanied by the destruction of one hexagonal ring, the time scale for destruction of a typical 19 ring PAH molecule would be about $10^{6} \mathrm{yr}$ in the models. This lifetime is comparable to the longest equilibrium time scale for formation and destruction of $\mathrm{H}_{2}, 5 \times 10^{6} \mathrm{yr}$ in the centers of our cloud models; therefore the rates of these reactions represent the maximum rates that are in harmony with the assumption of steady state.

Because the dissociative recombination of $\mathrm{H}_{3}{ }^{+}$and $\mathrm{H}_{2} \mathrm{D}^{+}$is slow, we include the reactions

$$
\begin{aligned}
\mathrm{H}_{3}^{+}+\mathrm{PAH} & \rightarrow \mathrm{PAH}^{+}+\mathrm{H}+\mathrm{H}_{2} \\
& \rightarrow \mathrm{PAH}^{+}+\mathrm{H}+\mathrm{H}+\mathrm{H} \\
\mathrm{H}_{3}{ }^{+}+\mathrm{PAH}^{-} & \rightarrow \mathrm{PAH}+\mathrm{H}+\mathrm{H}_{2} \\
& \rightarrow \mathrm{PAH}+\mathrm{H}+\mathrm{H}+\mathrm{H}
\end{aligned}
$$

with total rate coefficients of respectively $4.4 \times 10^{-9} \mathrm{~cm}^{3} \mathrm{~s}^{-1}$ and $1.3 \times 10^{-7}(10 / T)^{1 / 2} \mathrm{~cm}^{3} \mathrm{~s}^{-1}$ (Omont 1986) and equal branching ratios for the various channels. Similar reactions occur for $\mathrm{H}_{2} \mathrm{D}^{+}$.

We have incorporated into the chemical models of van Dishoeck and Black (1986) the atoms $\mathrm{Li}, \mathrm{Na}, \mathrm{Mg}, \mathrm{Al}, \mathrm{Si}, \mathrm{P}, \mathrm{S}, \mathrm{K}$, $\mathrm{Ca}, \mathrm{Ti}, \mathrm{Cr}, \mathrm{Mn}, \mathrm{Fe}, \mathrm{Co}, \mathrm{Ni}, \mathrm{Zn}$, and $\mathrm{Rb}$. They are all ionized by the interstellar radiation field and $\mathrm{Ca}$ is partly doubly ionized. The photoionization rates were calculated using the methods of Roberge, Dalgarno, and Flannery (1981). We adopted recombination rate coefficients similar to those listed by Péquignot and Aldrovandi (1986). Charge transfer reactions of molecular ions with neutral metal atoms have been included (see Oppenheimer and Dalgarno 1974; Prasad and Huntress 1980). Detailed models have been worked out for the clouds toward $\zeta$ Persei and $\zeta$ Ophiuchi.

\section{MODELS}

For the $\zeta$ Persei cloud, we have explored the effects of large molecules on model F of van Dishoeck and Black (1986) which has a central density of $325 \mathrm{~cm}^{-3}$, a central temperature of 30 $\mathrm{K}$, and a radiation field enhanced by a factor of 3.5 for grain model 2. For the $\zeta$ Ophiuchi cloud, we have obtained results for models $B$ and $G$. Model $G$ has a central density of 350 $\mathrm{cm}^{-3}$, a central temperature of $30 \mathrm{~K}$, and a radiation field enhancement factor of 3.5 with grain model 2. Model $\mathrm{B}$ has a steeper density gradient with a central density of $800 \mathrm{~cm}^{-3}$ and a central temperature of $20 \mathrm{~K}$.

Figure 2 illustrates the variations of various species with depth into the $\zeta$ Persei model for a PAH abundance $x_{\mathrm{PAH}}=n_{\mathrm{PAH}} / n_{\mathrm{H}}=10^{-7}$, where $n_{\mathrm{PAH}}=n(\mathrm{PAH})+n\left(\mathrm{PAH}^{-}\right)$ $+n\left(\mathrm{PAH}^{+}\right)$, and $n_{\mathrm{H}}=n(\mathrm{H})+2 n\left(\mathrm{H}_{2}\right)$. PAH is mostly neutral at the edge of the cloud, whereas in the center of the cloud the $\mathrm{PAH}^{-}$abundance is comparable to that of PAH. The PAH ${ }^{+}$ abundance is an order of magnitude smaller. In comparing Figures 1 and 2, note that the relative amounts of PAH and $\mathrm{PAH}^{-}$depend on the ratio of ultraviolet intensity to total density and that this ratio has a larger value in the $\zeta$ Persei model. Tables 1 and 2 list the resulting column densities for various species in models with $\mathrm{PAH}$ abundances ranging from 0 to $10^{-5}$. In addition to the PAH reactions some reaction rate coefficients have been updated compared with the model of

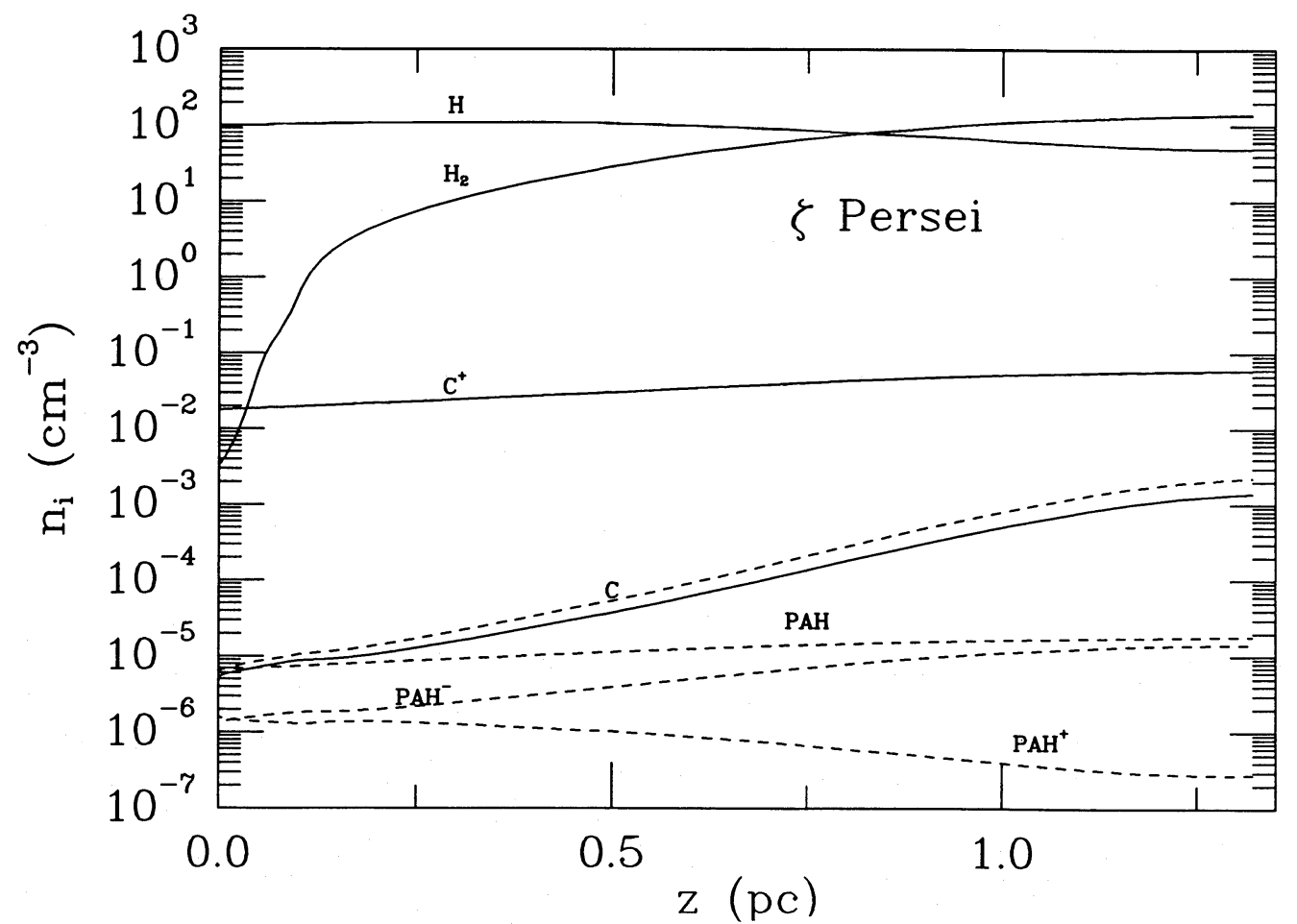

FIG. 2.-Depth variations of the concentrations of several species in model $\mathrm{F}$ of the $\zeta$ Persei cloud (see text). Solid curves show abundances for $x_{\mathrm{PAH}}=0$ while dashed curves indicate abundances for $x_{\mathrm{PAH}}=10^{-7}$. The presence of PAHs at the level of $x_{\mathrm{PAH}}=10^{-7}$ has no discernible effect on the abundance curves for $\mathrm{H}, \mathrm{H}_{2}$,
and $\mathrm{C}^{+}$. 


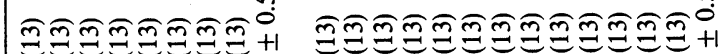

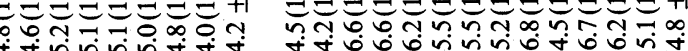

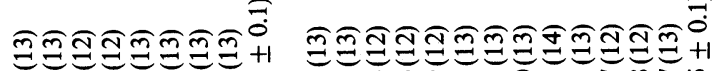

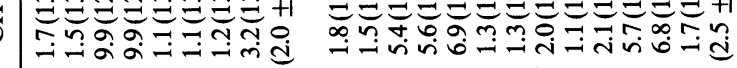

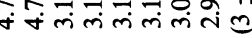

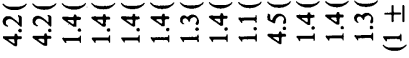

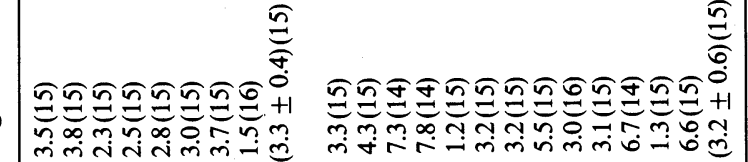

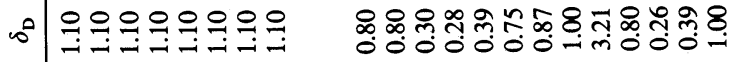

0

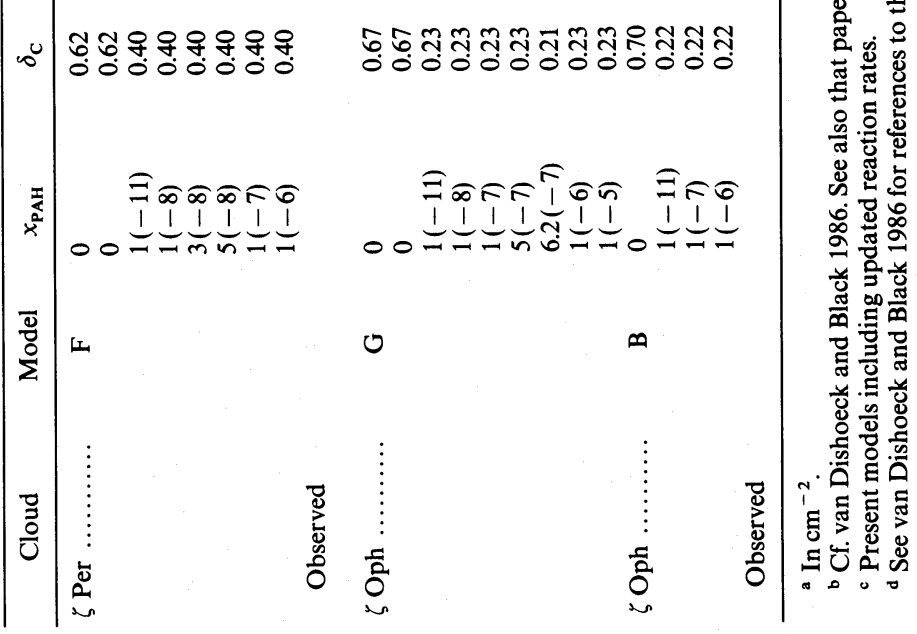


TABLE 2

Column Densities a and Ratios of Column Densities in the $\zeta$ Persei and $\zeta$ Ophiuchi Clouds

\begin{tabular}{|c|c|c|c|c|c|c|c|c|c|c|}
\hline Cloud & Model & $x_{\text {PAH }}$ & $N(\mathrm{Si})$ & $N(\mathrm{Al})$ & $N(\mathrm{Zn})$ & $N\left(\mathrm{C}^{+}\right) / N(\mathrm{C})$ & $N\left(\mathrm{Mg}^{+}\right) / N(\mathrm{Mg})$ & $N\left(\mathrm{~S}^{+}\right) / N(\mathrm{~S})$ & $N\left(\mathrm{Ca}^{+}\right) / N(\mathrm{Ca})$ & $N\left(\mathrm{Fe}^{+}\right) / N(\mathrm{Fe})$ \\
\hline \multirow[t]{6}{*}{$\zeta$ Per $\ldots \ldots \ldots \ldots \ldots$} & $\mathbf{F}$ & $1(-11)$ & $1.1(13)$ & $3.9(9)$ & $3.6(10)$ & 130 & 229 & 457 & 556 & 624 \\
\hline & & $1(-8)$ & $1.1(13)$ & $4.0(9)$ & $4.0(10)$ & 123 & 211 & 434 & 509 & 572 \\
\hline & & $3(-8)$ & $1.3(13)$ & $4.3(9)$ & $4.7(10)$ & 111 & 182 & 393 & 436 & 493 \\
\hline & & $5(-8)$ & $1.4(13)$ & $4.6(9)$ & $5.4(10)$ & 100 & 160 & 360 & 381 & 432 \\
\hline & & $1(-7)$ & $1.8(13)$ & $5.3(9)$ & $7.2(10)$ & 81.6 & 123 & 296 & 290 & 330 \\
\hline & & $1(-6)$ & $8.0(13)$ & $1.8(10)$ & $3.9(11)$ & 19.6 & 24.1 & 71.7 & 58.7 & 64.3 \\
\hline \multirow[t]{2}{*}{ Observed $^{b}$} & & & & & & 91 & 290 & 490 & 290 & 860 \\
\hline & & & & & & $63-135$ & $66-1700$ & $350-710$ & $180-440$ & $91-\infty$ \\
\hline \multirow[t]{7}{*}{$\zeta \mathrm{Oph} \ldots \ldots \ldots \ldots$} & G & $1(-11)$ & $1.9(11)$ & $2.2(9)$ & $2.0(10)$ & 196 & 277 & 683 & 849 & 814 \\
\hline & & $1(-8)$ & $2.0(11)$ & $2.3(9)$ & $2.3(10)$ & 183 & 249 & 639 & 760 & 729 \\
\hline & & $1(-7)$ & $3.5(11)$ & $3.2(9)$ & $4.7(10)$ & 115 & 131 & 398 & 393 & 386 \\
\hline & & $5(-7)$ & $1.0(12)$ & $7.4(9)$ & $1.5(11)$ & 43.6 & 42.1 & 148 & 129 & 126 \\
\hline & & $6.2(-7)$ & $1.1(12)$ & $8.2(9)$ & $1.8(11)$ & 39.1 & 36.1 & 131 & 114 & 110 \\
\hline & & $1(-6)$ & $1.8(12)$ & $1.3(10)$ & $2.8(11)$ & 25.0 & 23.0 & 83.8 & 72.8 & 69.2 \\
\hline & & $1(-5)$ & $1.4(13)$ & $9.2(10)$ & $2.0(12)$ & 3.8 & 2.8 & 11.0 & 10.3 & 8.9 \\
\hline \multirow[t]{3}{*}{$\zeta$ Oph $\ldots \ldots \ldots \ldots$} & B & $1(-11)$ & $2.1(11)$ & $3.5(9)$ & $2.0(10)$ & 211 & 225 & 715 & 514 & 700 \\
\hline & & $1(-7)$ & $4.6(11)$ & $5.8(9)$ & $5.4(10)$ & 106 & 90.9 & 366 & 199 & 281 \\
\hline & & $1(-6)$ & $2.7(12)$ & $2.6(10)$ & $3.4(11)$ & 20.3 & 14.6 & 68.3 & 33.9 & 45.4 \\
\hline \multirow[t]{2}{*}{ Observed $^{\mathbf{b}}$} & & & $<3.6(12)$ & $<1.6(10)$ & $<1.2(12)$ & 29 & 48 & 80 & 108 & 162 \\
\hline & & & & & & $13-260$ & $17-67$ & $52-124$ & $98-118$ & $40-280$ \\
\hline
\end{tabular}

NoTE.-In each case the heavy element abundances have been chosen to provide the best fit to observed column densities.

a In $^{-2}$.

b See van Dishoeck and Black (1986) and Black (1987) for references to the observations.

van Dishoeck and Black (1986), including an enhanced $\mathrm{CH}$ photoionization rate and an improved treatment of the $\mathrm{CO}$ photodissociation rate (van Dishoeck and Black 1988). As the tables show for $x_{\mathrm{PAH}}=0$, these changes have little effect on the abundances of the species of interest here.

The atomic column densities and ratios presented in Tables 1 and 2 are the best available values from the literature. In the cases of Mg II, Fe I, Si I, and S I, published column densities have been adjusted for improved oscillator strengths. Table 2 lists for the ratios of column densities both the value and the extreme range of values permitted by the published uncertainties. The full effects of unresolved velocity structure on curve of growth analyses remain to be explored.

The most sensitive probes of PAH chemistry in diffuse clouds appear to be the abundance ratios of neutral atoms and their first ions. Atomic ions that recombine slowly with electrons and react inefficiently with $\mathrm{H}_{2}$ can be removed more rapidly by PAHs when $x_{\mathrm{PAH}} \geq 10^{-7}$. Although the $\mathrm{C}^{+}$column density is poorly determined from observations of highly saturated lines, it appears from Tables 1 and 2 that the neutral fraction of carbon is in better agreement with observation if PAH reactions (or some other agent of enhanced recombination) are at work in the $\zeta$ Persei and $\zeta$ Ophiuchi clouds. This suggestion is strengthened by the comparison of models with upper limits on neutral atomic species for which the corresponding ions are well-measured ( $\mathrm{Si}, \mathrm{Al}, \mathrm{Zn})$ and with measured ratios of column densities for neutral atom-first ion pairs (C, Mg, S, Ca, and Fe). If PAHs have the chemical behavior we have assumed, then PAH abundances larger than $\sim 5 \times 10^{-7}$ and $\sim 2-3 \times 10^{-6}$ are excluded by the data for the $\zeta$ Persei and $\zeta$ Ophiuchi clouds, respectively. The atomic data for the $\zeta$ Ophiuchi cloud are inconsistent with the models if $x_{\text {PAH }}<10^{-7}$. For the $\zeta$ Persei cloud only the $\mathrm{Ca}^{+} / \mathrm{Ca}$ ratio is sufficiently well measured to exclude the possibility that $x_{\mathrm{PAH}} \approx 0$. Within the framework of the present models all of the data on atomic ionization in these clouds are reproduced well only if an additional recombining agent like the PAHs is acting to enhance the neutral fractions. Specifically, the systematic trends in atomic abundances shown in Table 2 are in harmony with $x_{\mathrm{PAH}} \approx 10^{-7}$ for the $\zeta$ Persei cloud and $x_{\mathrm{PAH}} \approx$ $6 \times 10^{-7}$ for the $\zeta$ Ophiuchi cloud. Note that in all cases the heavy element abundances have been chosen to provide the best fit to the observed column densities. In particular, the gas-phase carbon abundance is reduced compared with the models of van Dishoeck and Black (1986), if PAHs are included. An uncertainty of the order of a factor of 2 applies to the inferred PAHs abundances. This analysis can be viewed alternatively not as a demonstration of the existence of LMs or PAHs in diffuse clouds but as a restatement of a long-standing issue concerning diffuse clouds: that diagnostic probes like rotational excitation in molecules and fine-structure excitation in atoms indicate lower densities than do the atomic ionization equilibria (cf. van Dishoeck and Black 1986).

For the generic 50-atom PAH assumed here, the inferred abundance in the $\zeta$ Persei cloud would account for $1 \%$ of the reference abundance of carbon in all forms $([\mathrm{C} / \mathrm{H}]=$ $4.7 \times 10^{-4}$ in the Sun). The derived depletion factor $\delta_{\mathrm{C}}=$ 0.40 for the remainder of the gas-phase carbon still allows for almost $60 \%$ of the carbon to reside in grains. For the $\zeta$ Ophiuchi cloud $7 \%$ of the carbon is in PAHs, $21 \%$ in other gaseous forms, and $72 \%$ in grains.

Similar calculations can be performed for the $o$ Persei and $\chi$ Ophiuchi clouds. For the $o$ Persei cloud, a PAH abundance of a few times $10^{-7}$ would improve the comparison between the observational data on atomic species and the models. For the $\chi$ Ophiuchi cloud, the measurements are unfortunately too uncertain to make any inferences.

Our discussion of the ionization balance applies not only to PAHs, but to any other large molecule or small grain which is capable of neutralizing ions. The rate coefficients for charge exchange of ions with small grains of arbitrary shape are of the same order of magnitude as those adopted in this work with PAHs (Draine and Sutin 1987).

Some caution is appropriate. The models described here 
were constructed originally to reproduce as well as possible the available data on the abundance and excitation of $\mathrm{H}_{2}$, the abundances of other simple molecules, and the excitation of $\mathrm{C}_{2}$ and of atomic fine structure levels. Apart from the PAH reactions, other factors which influence the ionization balance are the temperature and density structure in the cloud, and the strength of the incident radiation field. Although these parameters have been constrained to reproduce a wide variety of observations, they are uncertain by a factor of 2 . The observed large molecular abundances suggest that it is unlikely that the density is much lower than the adopted values but it is possible that the density has been underestimated by a factor of 2 and the intensity of the radiation field somewhat overestimated. If so, the ionization balance would shift in favor of the neutral species, and the inferred upper limit on the PAH abundance would be lowered.

In order to examine the model-dependent effects of different density and temperature profiles, results are presented in Tables 1 and 2 for model B of the $\zeta$ Ophiuchi cloud, which has the highest central density of the models constructed by van Dishoeck and Black (1986). Within the factors of 2 uncertainty, the same LM/PAH abundance is inferred from the atomic ionization, as expected since all of the models were designed to have the same column-averaged ratios of ultraviolet absorption rates to gas density.

The PAHs have small effects on the abundances of observable molecules like $\mathrm{CH}, \mathrm{OH}$, and $\mathrm{HD}$ as long as $x_{\mathrm{PAH}} \leq 10^{-7}$. For higher PAH abundances, there is a competition between the reduction in the concentrations of major ions that initiate molecule formation by reactions with $\mathrm{PAH}^{-}$and the enhancement in the production of $\mathrm{H}_{2} \mathrm{O}$ and $\mathrm{CH}_{4}$ by the assumed reactions of $\mathrm{C}$ and $\mathrm{O}$ with $\mathrm{PAH}^{-}$which lead to larger production rates of $\mathrm{OH}$ and $\mathrm{CH}$ by photodissociation. The predicted column densities of other molecules, such as $\mathrm{H}_{2} \mathrm{O}$ and $\mathrm{CN}$, approach or exceed the observed values only when $x_{\mathrm{PAH}}>10^{-6}$ for the $\zeta$ Ophiuchi cloud. When the depletion factor $\delta_{\mathrm{C}}$ is lowered for $x_{\mathrm{PAH}} \geq 10^{-7}$ in order to reproduce the column density of atomic carbon, the abundances of most carbon-bearing molecules are reduced as a result. A particularly interesting case is provided by the $\mathrm{CH}$ molecule. If the effects of PAHs on the ionization balance are taken into account but the reactions (11)-(12) of $\mathrm{C}$ and $\mathrm{H}$ with PAH and $\mathrm{PAH}^{-}$to form $\mathrm{CH}_{4}$ are not included in the models, then the $\mathrm{CH}$ column density falls below the observed values by factors of $2-4$. The discrepancy could be remedied with a corresponding increase in the rate coefficient of radiative association

$$
\mathrm{C}^{+}+\mathrm{H}_{2} \rightarrow \mathrm{CH}_{2}^{+}+h v
$$

above the adopted value of $7 \times 10^{-16} \mathrm{~cm}^{3} \mathrm{~s}^{-1}$; however, such a rate would begin to conflict with the upper limit of $1.5 \times 10^{-15} \mathrm{~cm}^{3} \mathrm{~s}^{-1}$ (Luine and Dunn 1985) measured at 10 $\mathrm{K}$. On the other hand, with the inclusion of the reactions with $\mathrm{PAH}$ and $\mathrm{PAH}^{-}$, the production of $\mathrm{CH}_{4}$ is large enough that its subsequent photodissociation provides a major source of $\mathrm{CH}$ and the $\mathrm{CH}$ abundance remains consistent with observations at $x_{\mathrm{PAH}} \approx 10^{-7}-10^{-6}$ without any other compensating changes in the carbon chemistry.

The abundances of oxygen-bearing species such as $\mathrm{OH}$ are not much affected by the presence of PAHs in the cloud, as Table 1 illustrates. Although the $\mathrm{H}^{+}$column density is reduced considerably by charge transfer for $x_{\mathrm{PAH}} \geq 10^{-7}$, the $\mathrm{H}_{3}{ }^{+}$ column density remains nearly constant. The abundance of $\mathrm{H}_{3}{ }^{+}$is governed primarily by cosmic-ray ionizations and reactions with oxygen-bearing neutral species. The principal initi- ating reaction in the oxygen chain is the $\mathrm{O}+\mathrm{H}_{3}{ }^{+}$reaction to form $\mathrm{OH}^{+}$and the abundances of product molecules, including $\mathrm{OH}$ and $\mathrm{CO}$, are not much changed.

In contrast, the HD column density decreases strongly with increasing $\mathrm{PAH}$ abundance mainly because of the large reduction of both the $\mathrm{H}^{+}$and $\mathrm{D}^{+}$concentrations through charge-transfer with PAHs, which reduces the rates of formation of $\mathrm{HD}$ by the $\mathrm{H}^{+}+\mathrm{D} \rightarrow \mathrm{H}+\mathrm{D}^{+}$reaction followed by $\mathrm{D}^{+}+\mathrm{H}_{2} \rightarrow \mathrm{HD}+\mathrm{H}^{+}$. The entries for $\zeta$ Persei in Table 1 illustrate the effect of PAH reactions on the HD column density for a fixed deuterium abundance ratio, $[\mathrm{D} / \mathrm{H}]=1.5$ $\times 10^{-5} \delta_{\mathrm{D}}$. The entries for $\zeta$ Ophiuchi show how the abundance must be increased with increasing $x_{\mathrm{PAH}}$ in order to reproduce the observed column density of HD. The analysis of the HD abundance involves two disposable parameters, $\delta_{\mathrm{D}}$ and the cosmic-ray ionization rate. The latter is also constrained by the abundance of $\mathrm{OH}$, for which the chemistry is rather well understood. For any deuterium abundance $\delta_{\mathrm{D}} \leq 1.0$, the results of Table 1 and the observed HD column densities imply $x_{\mathrm{PAH}}<6 \times 10^{-7}$ for $\zeta$ Persei and $x_{\mathrm{PAH}}<10^{-6}$ for $\zeta$ Ophiuchi. These limits are virtually the same as those provided by the atomic data. Aside from the remaining uncertainties in the inferred cosmic-ray ionization rates, the implied deuterium abundances agree within $25 \%$ for $x_{\mathrm{PAH}}=10^{-7}$ in $\zeta$ Persei and $x_{\mathrm{PAH}}=6.2 \times 10^{-7}$ in $\zeta$ Ophiuchi. The HD abundance could also be increased in the models by lowering the line widths in the calculation of the HD photodissociation rates, but the HD column densities inferred from the measured equivalent widths would be increased and the discrepancy would persist.

The postulated reactions of atomic species with PAHs to form molecules such as $\mathrm{CH}_{4}$ and $\mathrm{NH}_{3}$ open a pathway for the synthesis of complex molecules in diffuse clouds. In particular, the predicted abundances of related species such as $\mathrm{H}_{2} \mathrm{CO}$, $\mathrm{CH}_{3}$, and $\mathrm{C}_{2} \mathrm{H}_{2}$ are enhanced by several orders of magnitude if PAHs are included. In somewhat thicker and denser clouds than those considered here, the predicted amounts of $\mathrm{H}_{2} \mathrm{CO}$ may be observable.

Regardless of the interpretation of the ad hoc chemistry introduced here in the guise of large molecules, the model analysis provides an internally consistent set of semiempirical corrections for unseen stages of ionization in diffuse clouds. This is crucial for the determination of gas-phase abundances from observations of a rare form of an element such as $\mathrm{Li}, \mathrm{Na}$, $\mathrm{K}$, or $\mathrm{Rb}$ that is expected to exist predominantly in an unobservable stage of ionization. In Table 3 the element abundances deduced from the best available observations and the bestfitting ionization models are collected. The results in Table 3 for depletions are in qualitative agreement with values elsewhere in the leterature. The inferred depletions are more severe for $\zeta$ Ophiuchi than for $\zeta$ Persei, with the exceptions of Ti and $\mathrm{Mn}$. We stress that detailed ionization models are required to correct element abundances for unseen stages of ionization. Empirical corrections are often used in the literature; for example, the observed ratio of calcium column densities, $N\left(\mathrm{Ca}^{+}\right) / N(\mathrm{Ca})$, is taken to characterize the ratio of the rates of ionization $\Gamma$ and recombination $\alpha$

$$
N\left(\mathrm{Ca}^{+}\right) / N(\mathrm{Ca}) \approx n\left(\mathrm{Ca}^{+}\right) n(\mathrm{Ca})=\Gamma(\mathrm{Ca}) /[\alpha(\mathrm{Ca}) n(e)] .
$$

With adjustments for different photoionization cross sections and recombination rate coefficients, this ratio is applied to the ionization balance in lithium or sodium, for example, for which only the neutral species are observed. This procedure is strictly applicable only if the ratios of local densities do not vary significantly with depth. Our detailed models for $\zeta$ Persei show 
TABLE 3

INFERRED GAS-PHASE ABUNDANCES

\begin{tabular}{|c|c|c|c|c|}
\hline \multirow{2}{*}{$\begin{array}{c}\text { ELEMENT } \\
\mathrm{X}\end{array}$} & \multicolumn{2}{|c|}{$\zeta$ ОРн } & \multicolumn{2}{|c|}{$\zeta$ PER } \\
\hline & $n_{\mathrm{X}} / n_{\mathrm{H}}$ & $\delta_{\mathrm{x}}$ & $n_{\mathrm{X}} / n_{\mathrm{H}}$ & $\delta_{\mathbf{X}}$ \\
\hline PAH & $6(-7)$ & & $1(-7)$ & \\
\hline $\mathbf{L i} \ldots$ & $3.5(-10)$ & $1.6(-1)$ & $6.2(-10)$ & $2.8(-1)$ \\
\hline $\mathrm{C}, \ldots \ldots \ldots \ldots \ldots$ & $9.7(-5)$ & $2.1(-1)$ & $1.9(-4)$ & $4.0(-1)$ \\
\hline $\mathrm{Na} \ldots \ldots \ldots \ldots \ldots$ & $7.6(-7)$ & $3.6(-1)$ & $2.1(-6)$ & 1.0 \\
\hline $\operatorname{Mg} \ldots \ldots \ldots \ldots \ldots$ & $5.0(-6)$ & $1.2(-1)$ & $5.5(-6)$ & $1.2(-1)$ \\
\hline $\mathrm{Al} \quad \ldots \ldots \ldots \ldots \ldots$ & $7.6(-9)$ & $2.5(-3)$ & 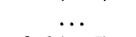 & . \\
\hline $\mathrm{Si} \quad \ldots \ldots \ldots \ldots \ldots$ & $8.2(-7)$ & $1.9(-2)$ & $2.6(-5)$ & $6.1(-1)$ \\
\hline $\mathrm{P} \quad \ldots \ldots \ldots \ldots \ldots+\cdots$ & $2.2(-8)$ & $7.8(-2)$ & $3.8(-4)$ & $1.4(-1)$ \\
\hline $\mathrm{S} \ldots$ & $8.3(-6)$ & $4.9(-1)$ & $1.2(-5)$ & $6.9(-1)$ \\
\hline $\mathbf{K} \ldots$ & $1.0(-8)$ & $7.8(-2)$ & $3.7(-8)$ & $2.9(-1)$ \\
\hline $\mathrm{Ca} \quad \ldots \ldots \ldots \ldots \ldots$ & $6.0(-10)$ & $2.7(-4)$ & $8.5(-10)$ & $3.9(-4)$ \\
\hline Ti $\ldots \ldots \ldots \ldots \ldots \ldots$ & $2.3(-10)$ & $1.9(-3)$ & $5.6(-11)$ & $4.7(-4)$ \\
\hline Mn .. & $1.5(-8)$ & $5.9(-2)$ & $8.6(-9)$ & $3.3(-2)$ \\
\hline $\mathrm{Fe} \quad \ldots \ldots \ldots \ldots \ldots$ & $3.2(-8)$ & $7.5(-4)^{a}$ & $3.6(-7)$ & $8.3(-3)$ \\
\hline $\mathrm{Ni}, \ldots \ldots \ldots \ldots \ldots$ & $9.9(-9)$ & $5.8(-3)$ & $\cdots$ & $\cdots$ \\
\hline Zn $\ldots \ldots \ldots \ldots \ldots$ & $1.4(-8)$ & $3.6(-1)$ & $\ldots$ & $\ldots$ \\
\hline $\mathrm{Rb} \ldots \ldots \ldots \ldots \ldots$ & $<7.2(-11)$ & $<1.8(-1)$ & $<7.5(-11)$ & $<1.9(-1)$ \\
\hline
\end{tabular}

${ }^{\text {a }} \mathrm{Fe}$ abundance adjusted to $\mathrm{Fe} \mathrm{I}$ column density. that the local ratio of concentrations, $n(\mathrm{Li}) / n(\mathrm{Na})$, increases by a factor of 2.6 from the edge to the center of the cloud, while the density ratio, $n\left(\mathrm{Ca}^{+}\right) / n(\mathrm{Ca})$, decreases from 4800 at the edge to 160 at the center. This example of depth dependent ionization suggests that empirical ionization corrections based on ratios of column densities can be inaccurate. Based on the present ionization models of $\zeta$ Persei and $\zeta$ Ophiuchi, the abundance of interstellar lithium in the gas phase is found to be 3-5 times larger than the primordial abundance $[\mathrm{Li} / \mathrm{H}]=(1.1 \pm 0.4) \times 10^{-10}$ derived by Spite and Spite (1982). The depletion factors for lithium in Table 3 refer to the abundance, $[\mathrm{Li} / \mathrm{H}]=2.2 \times 10^{-9}$, in carbonaceous chondrites.

This work has been supported by grant AST-86-17675 to Harvard University by the National Science Foundation, Division of Astronomical Sciences, by the NASA Astrophysical Theory Program through grant NAGW-763 to the University of Arizona, and by the National Science Foundation through grant RII-86-20342 to Princeton University. E. F. v D. and J. H. B. acknowledge the hospitality of the Institute for Advanced Study, Princeton, where part of this work was carried out, and its support through NSF grant PHY 86-20266. They also enjoyed stimulating discussions with B. T. Draine.

\section{REFERENCES}

Allamandola, L. J., Tielens, A. G. G. M., and Barker, J. R. 1985, Ap. J. (Letters), 290, L25.

Black, J. H. 1987, in Spectroscopy of Astrophysical Plasmas, ed. A. Dalgarno and D. Layzer (Cambridge: Cambridge University Press), p. 279.

Crawford, M. K., Tielens, A. G. G. M., and Allamandola, L. J. 1985, Ap. J. (Letters), 293, L45.

d'Hendecourt, L. B., and Léger, A. 1987, Astr. Ap., 180, L9.

Draine, B. T. 1978, Ap. J. Suppl., 36, 595.

Draine, B. T., and Sutin, B. $1987, A$ p. J., 320, 803

Duley, W. W. 1986, Quart. J.R.A.S., 27, 403.

Duley, W. W., and Williams, D. A. 1981, M.N.R.A.S., 186, 269. .1986, M.N.R.A.S., $219,859$.

Léger, A., and d'Hendecourt, L. B. 1985, Astr. Ap., 146, 81.

\author{
Léger, A., and Puget, J. L. 1984, Astr. Ap. 137, L5. \\ Lepp, S., and Dalgarno, A. 1988, Ap. J., 324, 553. \\ Luine, J. A., and Dunn, G. H. 1985, Ap. J. (Letters), 299, L67. \\ Omont, A. 1986, Astr. Ap., 164, 159. \\ Oppenheimer, M., and Dalgarno, A. 1974, Ap. J., 192, 29. \\ Péquignot, D., and Aldrovandi, S. M. V. 1986, Astr. Ap., 161, 169 \\ Prasad, S. S., and Huntress, W. T. 1980, Ap. J. Suppl., 43, 1. \\ Roberge, W. G., Dalgarno, A., and Flannery, B. P. 1981, Ap. J., 243, 817. \\ Spite, F., and Spite, M. 1982, Astr. Ap., 115, 357. \\ van der Zwet, G. P., and Allamandola, L. J. 1985, Astr. Ap. 146, 76 \\ van Dishoeck, E. F., and Black, J. H. 1986, Ap. J. Suppl., 62, 109. \\ 1988 , in preparation. \\ van Dishoeck, E. F., and Dalgarno, A. 1984, Ap. J., 277, 576.
}

J. H. BLACK : Steward Observatory, University of Arizona, Tucson AZ 85721

A. Dalgarno and S. LePp: Harvard-Smithsonian Center for Astrophysics, 60 Garden Street, Cambridge MA 02138

E. F. VAN Dishoeck : Princeton University Observatory, Peyton Hall, Princeton NJ 08544 